\title{
Intestinal perfusion of dietary levels of aluminium: association with the mucosa
}

\author{
J J Powell, C C Ainley, R Evans, R P H Thompson
}

\begin{abstract}
An aluminium $(93 \mu M)$ sulphate solution freshly adjusted to $\mathrm{pH} 7 \cdot 0$ was perfused through the rat small bowel to mimic the reported physiological conditions that follow dietary aluminium ingestion. One third of this aluminium was taken up from the perfusate, but $>90 \%$ of this was then recovered from the intestinal mucus/mucosa and most $(>70 \%)$ from the distal third of the small bowel. The fresh perfusate was shown by ultrafiltration to contain largely particulate/colloidal aluminium-hydroxide, and this probably adhered to intestinal mucus which may be an important barrier to the gastrointestinal absorption of aluminium.

(Gut 1994; 35: 1053-1057)
\end{abstract}

The toxicity of aluminium is now well recognised, and occurs at even low levels in plants, ${ }^{1}$ fish, ${ }^{2}$ and human cells. ${ }^{3}$ Encephalopathy, bone disease, and anaemia have been reported in patients with impaired renal excretion of aluminium, ${ }^{4}$ particularly those who are exposed to the metal in dialysis water. The efficiency of renal excretion and impermeability of the intestine to aluminium are sufficient to prevent acute toxicity in normal subjects, although not all absorbed aluminium is necessarily excreted, and long term loading may occur even in the normal population. ${ }^{5}$

It is therefore important to understand the mechanisms of the intestinal absorption of typical low dietary levels of aluminium. Previous studies have considered only unphysiologically high levels and the chemistry of aluminium is such that the results cannot be extrapolated to much lower dietary conditions. ${ }^{6}$

The average UK daily intake of aluminium is around $8 \mathrm{mg},{ }^{7}$ diluted by food plus 1.5 litres fluid, all matched by a similar volume of endogenous secretions during ingestion and transit. Thus, the concentration of aluminium in the intestinal lumen is about $8 \mathrm{mg}$ in 4 litres or $75 \mu \mathrm{mol} / 1$, although clearly this derived estimate is highly variable. At such a concentration, aluminium is expected to precipitate at the near neutral $\mathrm{pH}^{8}$ found in the small bowel, and Partridge et $a l^{9}$ showed that at much higher concentrations aluminium will indeed precipitate in the intestinal lumen. This has therefore been proposed as one limiting factor in the gastrointestinal absorption of the much lower, physiological concentrations of dietary aluminium. ${ }^{10-12}$ However, a large intestinal uptake of both soluble and precipitated aluminium by the bowel, but not its systemic transfer, has been consistently demonstrated, ${ }^{13-15}$ showing that retention in the mucosa, rather than luminal precipitation, is the major limiting factor in absorption. This could be due to either mucosal ${ }^{13}$ or extramucosal factors, such as the mucus layer. ${ }^{7}$ These studies ${ }^{13-15}$ again used supraphysiological levels of aluminium.

The aim of this study was to develop an in situ rat gut perfusion technique to study the fate of dietary levels of aluminium, and to see whether such physiological quantities of the metal also associate with the intestinal mucosa.

\section{Methods}

PREPARATION OF PERFUSATE

A solution of $50 \mathrm{mM} 4$-morpholinepropanesulphonic acid (MOPS) buffered saline was prepared at $\mathrm{pH} 7 \cdot 0$. Immediately before each experiment an aliquot of aluminium sulphate (pH 2.5, stock solution) was added to the MOPS buffer to yield an isotonic solution at $\mathrm{pH} 7 \cdot 0$ containing $93 \mu \mathrm{M}$ aluminium.

In preliminary experiments, this solution was thoroughly mixed by shaking, and precipitation was then allowed to ensue. The precipitate was not visible by inspection, but was confirmed under laser light (Malvern autosizer 2C). The precipitate remained as a suspension and did not significantly alter under the laser light for at least 15 hours, both at room temperature and at $37^{\circ} \mathrm{C}$.

The fresh perfusate was maintained in virgin polypropylene containers (Nalgene; BDH Ltd) that had been previously acid washed $(0 \cdot 32 \mathrm{M}$ nitric acid $/ 24$ hours) and then soaked for 24 hours, twice, in ultrapure water (Elga UHP). The concentration of total aluminium in aliquots of the fresh perfusate was confirmed by analysis with inductively coupled plasma optical emission spectrometry (ICPOES) as below. The concentration of aluminium remaining in solution in the freshly prepared perfusate was assessed with precleaned Centricon-10 (10000 molecular weight cut-off, Amicon Ltd) ultrafiltration devices. Results were compared to similar solutions containing $7 \cdot 4 \mu \mathrm{M}$ aluminium, also ultrafiltered through precleaned devices. These were precleaned by centrifuging $2 \mathrm{ml}$ sodium hydroxide $(0 \cdot 1 \mathrm{M})$ and then $2 \mathrm{ml}$ MOPS buffered saline ( $\mathrm{pH} 7 \cdot 0$ ) through the ultrafilters, according to the manufacturer's instructions. 
PERFUSION TECHNIQUE

Ten male Wistar rats $(300-350 \mathrm{~g})$ were fasted without coprophagy overnight (food withdrawn for 12 hours) and then anaesthetised (five further experiments were discarded; see 'Perfusion system'). Two cannulae were inserted, one immediately distal to the ligament of Treitz and the other proximal to the ileo-caecal junction. The gut was perfused with an isotonic aluminium-free MOPS/saline solution $(\mathrm{pH} 7.0$ ) for 10 minutes at $0.4 \mathrm{ml} / \mathrm{min}$, and then with air at the same rate until the bowel was clear of all perfusate. The whole small intestine was then perfused in situ, using a different and specially prepared single pass perfusion system (see below), with aluminium (93 $\mu \mathrm{M}$ total) in MOPS buffered saline at $37^{\circ} \mathrm{C}$ and $0.4 \mathrm{ml} / \mathrm{min}$ for 40 minutes. After this, residual luminal perfusate was removed by perfusing with air and the animal was killed. The whole small bowel was excised and divided into three segments of equal length (proximal; middle; distal). Sections (3-4 mm) of bowel tissue were taken from the proximal end of each section of bowel for histological examination and comparison with similar sections of bowel taken from non-perfused rats. In the first six experiments the mucus and mucosa were scraped off each of the three segments using an acid-washed perspex slide and were then weighed; for the last four experiments the mucus was removed by squeezing the bowel evenly along its length, and again the collected mass was weighed. Sections of the squeezed and scraped bowel were also taken for histological examination and assessment of loss of the mucus/mucosa.

Two animals were perfused as above, but with aluminium-free MOPS saline buffer, and the scraped mucus and mucosa were similarly collected, digested, and analysed for aluminium.

All sections of bowel were fixed in formalin, processed for wax sections, and stained with haematoxylin and eosin for light microscopy.

\section{PERFUSION SYSTEM}

Warmed perfusate $\left(45^{\circ} \mathrm{C}\right)$ was maintained in a closed polypropylene reservoir and drawn through polyethylene tubing $(4 \mathrm{~mm}$ internal diameter) with a constant pump delivery rate. This was passed through a heating coil $\left(42^{\circ} \mathrm{C}\right)$, and then a plastic three way tap immediately before delivery to the proximal bowel via a glass cannula. The final perfusate temperature was $37^{\circ} \mathrm{C}$ and the flow rate was $0.4 \mathrm{ml} / \mathrm{min}$. The perfusate from the distal bowel was collected through a glass cannula and teflon tube into an acid washed polypropylene container. The perfused bowel was kept moist with a saline $\left(37^{\circ} \mathrm{C}\right)$ soaked tissue on the serosal side and maintained at $37^{\circ} \mathrm{C}$ with an overhead heating lamp, thermostatically controlled by a probe on the serosa of the perfused bowel. Two important features of this system to minimise contamination or adhesion of aluminium were firstly, keeping the overall length of tubing (150 cm including the coil) to a minimum (which also reduces heat loss from the perfusate) and secondly, the use of polyethylene tubing except for the glass cannulae and heating coil. In addition, the system was acid washed (1.6M $\mathrm{HNO}_{3}$ Aristar grade) for one hour by perfusion, and then for one hour with ultrapure water. The aluminium containing perfusate was then passed through the system for two hours to allow aluminium to equilibrate with the tubing.

One perfusion experiment was performed each day, but the preparation was discarded if luminal blockage could not be cleared or if intestinal swelling rather than peristalsis was noted during perfusion; thus five preparations were discarded before the 10 successful experiments were completed.

\section{ANALYSES}

The scraped mucus and mucosa were collected into acid washed polypropylene tubes, weighed, and digested with $1 \cdot 5-3 \mathrm{ml}$ concentrated nitric acid (11.2 M: Aristar grade $\mathrm{BDH}$ Ltd) for 72 hours at $40^{\circ} \mathrm{C}$. This digestate was then diluted with 3-6 ml ultrapure water. The perfusate from the bowel was collected quantitatively into a similar container and acidified with $0.6 \mathrm{M}$ nitric acid before analysis for sodium, sulphur, and aluminium. Samples of perfusate from the first six animals were also collected for analysis of aluminium just before entering the bowel from the three way tap at the beginning and end of the perfusion period.

Analyses were performed in duplicate by simultaneous ICPOES at $308.21 \mathrm{~nm}$ for aluminium, $588.99 \mathrm{~nm}$ for sodium, and 180.67 for sulphur, using a Philips PV8050 spectrometer. Spiking and recovery experiments showed a linear response and full recovery for all three elements.

The squeezed mucus samples from the proximal, middle, and distal bowel were frozen at $-70^{\circ} \mathrm{C}$, before being thawed and analysed for protein content by sodium dodecyl sulphate-polyacrylamide gel electrophoresis. Standards of myoglobin (MW 18000), ovalbumin (MW 43000 ), albumin (MW 67 000), transferrin (MW 77000 ) and lactoferrin, which has a similar molecular weight to transferrin but a slightly lower mobility, were also run on the gel.

\section{Results}

ULTRAFILTERABLE ALUMINIUM

Only $14.3(1.3) \%$ of aluminium (13.3 (1.2) $\mu \mathrm{M}$, mean (SD)) from freshly prepared perfusate solution $(93 \mu \mathrm{M})$ was filterable through the Centricon-10 membrane ultrafilter $(n=4)$; but at the lower concentration of $7 \cdot 4 \mu \mathrm{M}, 94 \cdot 4$ $(6 \cdot 3) \%$ aluminium was recovered through the same filters and in the same solution at $\mathrm{pH} 7 \cdot 0$ $(n=4)$.

\section{HISTOLOGY}

The typical light microscopic histological appearance of the bowel mucosa immediately after perfusion is shown in Figure 1 . 


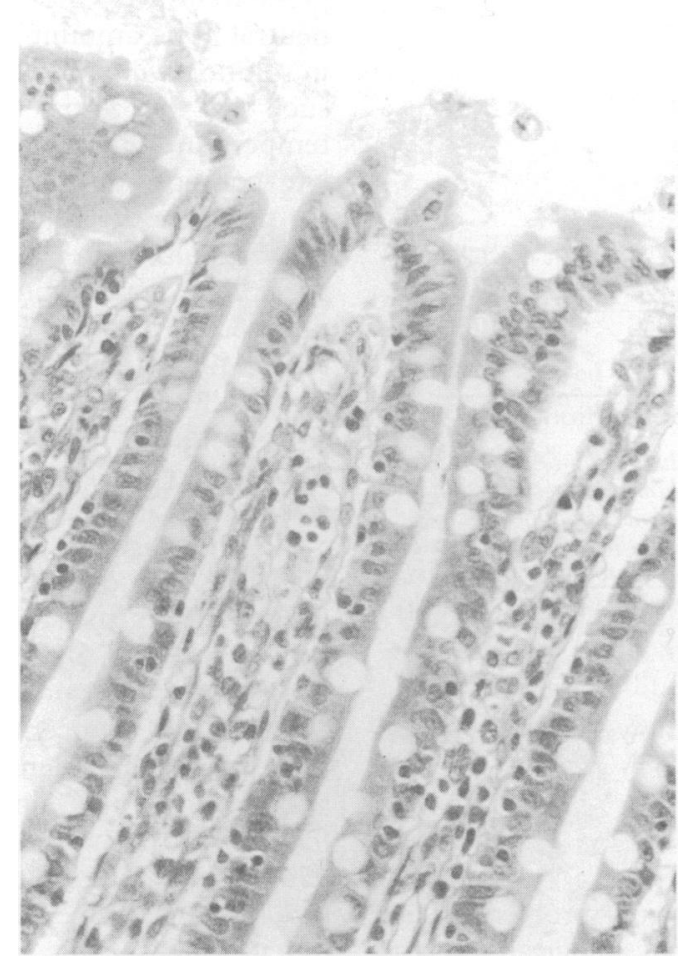

Figure 1: Light microscopy section (original magnification $\times 400$ ) showing villi of the rat small bowel following successful perfusion; shedding of the tip of the middle villus is seen.

Compared with non-perfused control tissue, the mucosa was normal, except for some additional sloughing of the villus tips (Fig 1). The mucosal scrape removed all mucosa, with a small amount of submucosa remaining on the muscle layer. The squeezed mucosa was intact (Fig 2), but occasionally some luminal mucus was still present indicating that squeezing did not completely remove all the mucus.

\section{PERFUSATE CONCENTRATIONS}

Once aluminium-containing perfusate had been equilibrated with the perfusion apparatus, there was no further loss of aluminium from the perfusate by adhesion to the system (or sodium from the sodium chloride or sulphur from the MOPS buffer). In contrast, the percentage change in the perfusate concentrations of aluminium, sodium, and sulphur after perfusion through the small bowel are shown in Figure 3 (one sulphur atom is present in one molecule of MOPS buffer).

\section{ALUMINIUM RECOVERY}

Figure 4 shows the distribution of aluminium after perfusion. The total aluminium perfused was $1.48 \mu \mathrm{mol} /$ experiment, and of this 62.2 $(6 \cdot 1) \%$ (mean (SD)) was recovered in the effluent; $92.8(15.4) \%$ (mean (SD)) of the unrecovered $(37 \cdot 8 \%)$ perfused aluminium was detected in the mucus/mucosal scrape, of which $11 \cdot 2(4 \cdot 8) \%$ was in the proximal, $17 \cdot 5$ $(4 \cdot 8) \%$ in the mid, and $71.3(5 \cdot 3) \%$ in the distal segments $(\mathrm{p}<0.001$, Student's $t$ test; distal $v$ others). The total aluminium recovered, from perfusate plus all sections of

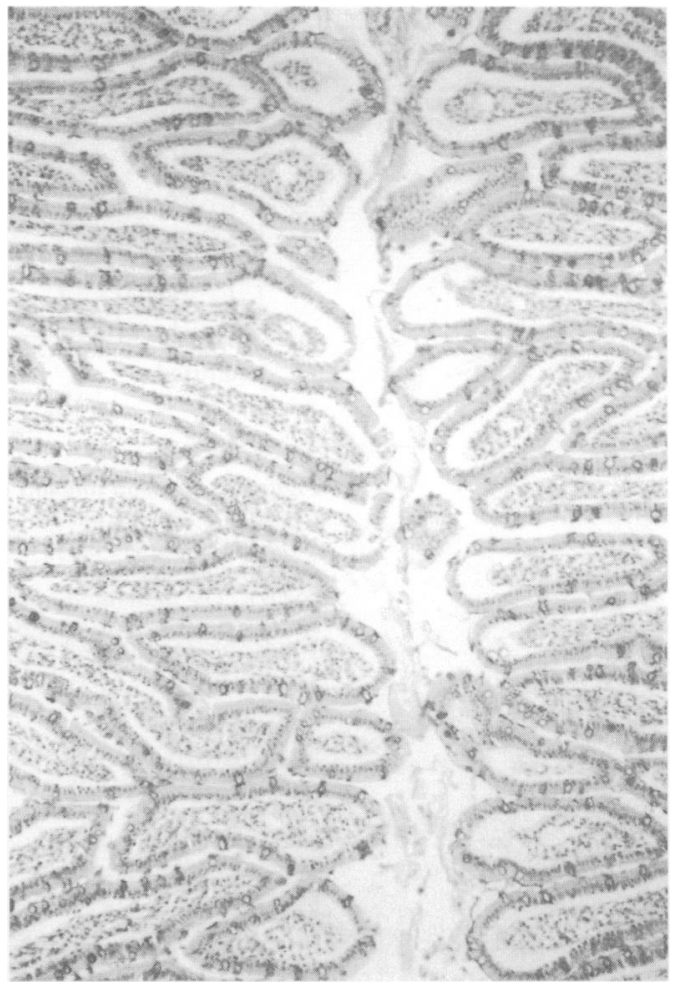

Figure 2: Light microscopy section (original magnification $\times 100$ ) showing the intestinal mucosa and lumen after removal of the luminal contents by squeezing the bowel. The mucosa remains intact but not all luminal contents are removed; the residual is mainly mucus.

the intestinal mucus/mucosa, was $97 \cdot 5(6 \cdot 4) \%$. Aluminium recovery from the mucus/mucosa of the two control perfusions was 0.008 and $0 \cdot 010 \mu \mathrm{mol}$ respectively.

\section{POLYACRYLAMIDE GELS}

Although in all 12 mucus specimens weak bands were seen for substances that ran with mobilities close to those of transferrin and lactoferrin, their intensities were the same in the three areas of the gut. In contrast, there were strong bands for a substance that migrated with the same mobility as albumin in all specimens of mucus from the proximal and middle gut, but not from any mucus of the distal gut. There were no protein bands that ran with the same intensity of distribution as

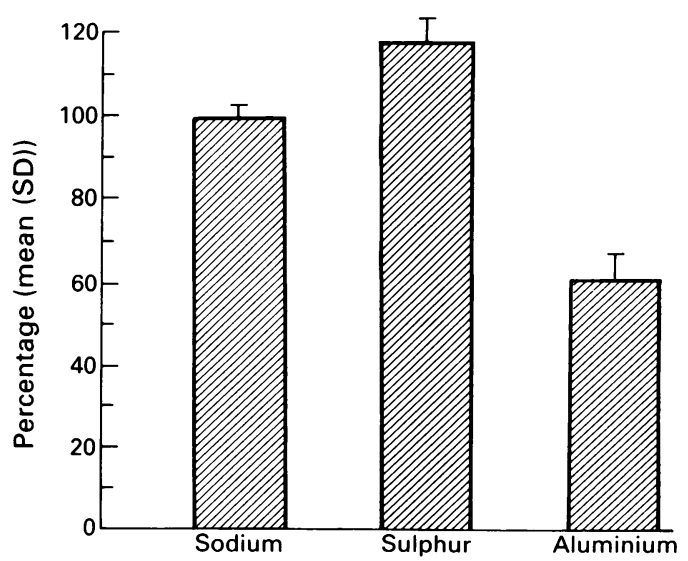

Figure 3: The concentrations of aluminium, sodium, and sulphur remaining in the perfusate after intestinal perfusion, as a percentage of their concentrations in the perfusate before intestinal perfusion $(n=6)$; mean $(S D))$. 


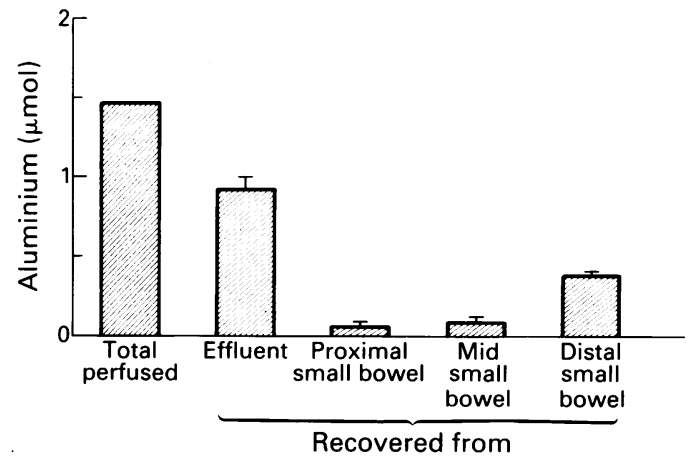

Figure 4: The total amount of aluminium ( $A l)$ perfused (Total $A l$ ) and the amount of aluminium recovered in the perfusate (Effluent) or the mucus/mucosa of the proximal, mid, and distal small bowel after perfusion $(n=6$; mean $(S D))$. The individual total aluminium recoveries (from effluent + total mucus/mucosa) of each rat were: $90 \cdot 7 \%$, $109 \%, 96 \cdot 1 \%, 99 \cdot 9 \%, 94.9 \%$, and $94 \cdot 3 \%$.

aluminium along the bowel, namely predominately distally.

\section{Discussion}

Contamination is a major problem when working with the low, physiological levels of aluminium, but may be excluded by rigorous acid washing and rinsing with deionised water or an appropriate buffer. We found that aluminium adheres to the perfusion apparatus, perhaps to the glass parts, and so the perfusate was allowed to pre-equilibrate with the system; the results then showed no change in the concentration of aluminium in the perfusate entering the bowel at the beginning and end of the experiment, and furthermore, did not differ from the concentration in the closed reservoir.

Peristalsis is an important physiological response to distension of the bowel, ${ }^{16}$ and when absent it indicates an ileus; such preparations (five) were therefore discarded. This seemed to be related to too high a perfusion rate or over handling of the bowel, and so handling was kept to a minimum and the perfusion rate to $0.4 \mathrm{ml} / \mathrm{min}$. We detected some sloughing of the villus tips even at this perfusion rate, and so it is surprising that at 25 times this rate no histological damage was reported by Van der Voet and de Woolf. ${ }^{17}$ Histological observations are important after perfusion of the bowel; for example aluminium could enter the tissue between cells ${ }^{18}$ of the damaged areas. Histological examination of squeezed bowel consistently showed that most of the mucus and no mucosa was collected; in addition, the absence of albumin in all distal samples of collected mucus showed that the samples were not significantly contaminated by plasma, interstitial, or cellular proteins. The mucus was not collected quantitatively and so it was not possible to assess its aluminium content.

Attempts to characterise experimental solutions containing aluminium have been made. ${ }^{1920} \mathrm{~A}$ buffer that does not bind aluminium should be used, and so phosphate buffers $^{20}$ should be avoided; we therefore used the non-interactive MOPS buffer. The aluminium sulphate dissociates when it dissolves yielding an acid $\mathrm{pH}$, but then precipitates as the $\mathrm{pH}$ is raised towards neutral. The amount of aluminium remaining in solution will depend on a number of factors, such as the initial aluminium compound, temperature, $\mathrm{pH}$, solution age, and electrolyte composition. ${ }^{21} \mathrm{~A}$ chelator may be used to prevent precipitation, if physiologically relevant, although it has been suggested that a precipitate may be physiological and occur in the normal bowel. ${ }^{9-12}$ We therefore characterised this by ultrafiltration to separate soluble from colloidal and particulate aluminium, and showed that in freshly prepared physiological solutions only about $14 \%$ of the aluminium was indeed 'in solution'.

After perfusion, the sodium concentration of the perfusate did not change, but sulphur, initially from the MOPS buffer, increased, probably because there is sulphur in bowel secretions. In contrast to these control elements, the concentration of aluminium in the perfusate fell significantly after perfusion, and of the total aluminium perfused only $62 \%$ was recovered in the collected perfusate. The retained aluminium was almost all $(92 \cdot 8$ $(15.4) \%$ ) recovered either on or in the mucus/mucosa, the apparent loss probably being partly due to errors in recovery at these low levels. Less than $1 \%$ of the total perfused aluminium would have been absorbed. ${ }^{7}$

The overall mucus/mucosal uptake of aluminium (38\%) far exceeded the initial total ultrafilterable aluminium $(14 \%)$ in the perfusate, showing that chiefly colloidal/ particulate aluminium hydroxide was taken up by the mucus/mucosa. The intramucosal uptake of macromolecules from the lumen is low, ${ }^{22}$ and it is therefore more likely that the freshly precipitated aluminium hydroxide, which is a fine positively charged floc, ${ }^{23}$ was adsorbed to negatively charged species of the surface mucosa or mucus. ${ }^{24} 25$ The distribution of such perfused aluminium down the bowel (Fig 4) suggests adhesion to mucus, since distally the number of goblet cells and mucus increases, ${ }^{26}$ while the surface area of the mucosa decreases. The aluminium may associate with mucus glycoprotein, ${ }^{25}$ because although other potential metal binding proteins were present in the collected mucus, these proteins did not correlate in their distribution along the bowel to the uptake of aluminium. Furthermore, such proteins could only specifically bind aluminium ions and not the particulate aluminium that was mainly perfused in these experiments.

In further work we shall therefore consider whether other forms of ingested aluminium at these dietary levels precipitate to form the hydroxide along the intestinal lumen, or whether the interaction with endogenous components such as mucus prevents precipitation. ${ }^{7}$ Nevertheless, we confirm that under these near normal conditions dietary levels of aluminium strongly associate with the intestinal mucus/ mucosa.

We thank Dr N Walsh, Royal Holloway and Bedford New College, for access to ICPOES and the Water Research Council, The Jean Shanks Foundation, London University Central Research Fund and the Special Trustees for St Thomas's Hospital for their support. 
1 Jackson ML. Aluminium of acid soils in the food chain and senility. Sci Total Environ 1983; 28: 269-76.

2 Birchall JD, Exley C, Chappell JS, Phillips MK. Acute toxicity of aluminium to fish eliminated in silicon-rich toxicity of aluminium to fish eliminate

3 Schofl C, Sanchez-Bueno A, Dixon CJ, Woods NM, Lee JAC, Cuthbertson KSR et al. Aluminium perturbs oscillatory phosphoinositide-mediated calcium signaling in hormone-stimulated hepatocytes. Biochem f 1990; 269: 547-50

4 Lindholm $\mathrm{T}$, Thysell $\mathrm{H}$, Ljunggren $\mathrm{L}$, Divino JC, Schunnesson $M$, Stenstam $M$. Aluminium in patients with uremia and patients with enteropathy. NierenHochdruckkr 1983; 12: 192-7.

5 Powell JJ, Thompson RPH. Aluminium deposition in bone after contamination of drinking water supply. Lancet 1990; ii: 888 (letter).

6 Ganrot PO. Metabolism and possible health effects of aluminium. Environ Health Perspect 1986; 65: 363-441.

7 Powell JJ, Thompson RPH. The chemistry of aluminium in the gastrointestinal lumen and its uptake and absorption. Proc Nutr Soc 1993; 5: 241-53.

8 Martin BR. The chemistry of aluminium as related to biology and medicine. Clin Chem 1986; 32: 1797-806. 9 Partridge N, Reigner F, White JL, Hem SL. Influence of dietary constituents on intestinal absorption of aluminium. Kidney Int 1989; 35: 1413-17.

10 Lote $\mathrm{CH}$, Saunders H. Aluminium: gastrointestinal absorption and renal excretion. Clin Sci 1991; 81: 289-95.

11 Stewart WK. Aluminium toxicity in individuals with chronic renal disease. In: (eds) Massey RC, Taylor D. Aluminium in food and the environment. London: Royal Society of Chemistry, 1989: 619.

12 Alfrey AC. Aluminium metabolism. Kidney Int 1986; 29 (Suppl 18): S8-11.

13 Cochran M, Goddard G, Ramm G, Ludwigson N, Marshall $\mathrm{J}$, Halliday J. Absorbed aluminium is found with two cytosolic protein fractions, other than ferritin, in the rat duodenum. Gut 1993; 34: 643-6.

14 Van der Voet GB, Van Ginkel MF, De Wolff FA. Intestinal absorption of aluminium in rats: stimulation by citric acid and inhibition by Dinitrophenol. Toxicol Appl Pharmacol 1989; 99: 90-7.

15 Feinroth M, Feinroth MV, Berlyne GM. Aluminium absorption in the rat everted gut sac. Miner Electrolyte Metab 1982; 8: 29-35.

16 Granger ND, Barrowman JA, Kvietys PR. The small intestine. In: Clinical gastrointestinal physiology: Philadelphia: WB Saunders Co, 1985: 141-207.

17 Van der Voet GB, de Wolff FA. The effect of di and trivalent iron on the intestinal absorption of aluminium in rats. Toxicol Appl Pharmacol 1987; 90: 190-7.

18 Froment $\mathrm{DH}$, Molitoris BA, Buddington B, Miller N, Alfrey AC. Site and mechanism of enhanced gastrointestinal absorption of aluminium by citrate. Kidney Int 1989; 36: 978-84.

19 Yokel RA, McNarmara PJ. Influence of renal impairment, chemical form and serum protein binding on intravenous and oral aluminium kinetics in the rabbit. Toxicol Appl Pharmacol 1988; 95: 32-43.

20 Lote CJ, Wood JA, Saunders HC. Renal filtration, reabsorption and excretion of aluminium in the rat. Clin Sci 1992; 82:

21 Chen DTY. Solubility products of aluminium hydroxide in various ionic solutions. Canadian fournal of Chemistry 1973; 51: 3528-33.

22 O'Hagan DT. Intestinal translocation of particulatesimplications for drug and antigen delivery. Advanced Drug Delivery Reviews 1990; 5: 265-85.

23 Arden TV. Water purification and recycling. In: Thompson $\mathrm{R}$. Ed. The modern inorganic chemicals industry. Special publication No 31. London: The Royal Society of Chemistry, 1982: 69-105.

24 Quaterman J. Metal absorption and the intestinal mucus layer. Digestion 1987; 37: 1-9.

25 Crowther RS, Marriott C. Counter-ion binding to mucus glycoproteins. I Pharm Pharmacol 1984; 36: 21-6.

26 Kulenkompff $\mathrm{H}$. The structural basis of intestinal absorption. In: Forth W, Rummel W. Eds. Pharmacology of intestinal absorption: gastrointestinal absorption of drugs. Vol 1; London: Pergamon, 1975: $1-69$. 